\title{
SOLAR MAGNETIC FIELDS AND CONVECTION
}

\author{
VII. A Review of the Primordial Field Theory \\ J. H. PIDDINGTON \\ National Measurement Laboratory, CSIRO, Sydney, Australia 2008
}

\begin{abstract}
We review the primordial field theory of solar magnetic fields (Papers I-VI) whose three main features are, first, a permanent dipole-like magnetic field, second a mainly toroidal field formed by shearing and rolling into individual, helically twisted ropes as suggested by Babcock, and third a mechanism for reversing the toroidal field. The theory explains numerous observational effects where the dynamo theory fails.

(i) An active region forms when a rope section emerges and expands layer by layer to form a rotating arch filament system and then spots. Only a rope model explains the radial inflow of magnetic elements to build up a spot, as well as the spiral structure and other features pointed out by Babcock. (ii) The model explains umbral and penumbral structures, the Wilson depression, Evershed flow, the sunspot energy deficit and the very slow loss of flux fragments by some sunspots. (iii) The model is then extended to background magnetic fields to show that surface magnetic fields are like the upppermost branches of a magnetic 'tree' whose trunk is a flux rope. This explains unipolar magnetic regions, 'pepper and salt', and ephemeral active regions. Tension in the submerged flux ropes accounts for the observed migrations of magnetic regions, active longitudes and magnetic longitudes. (iv) On a smaller scale spicules, mottles and other network elements are explained in terms of the tree structure. (v) The mechanism of reversal of the toroidal field system is explained and the manner of disposal of old toroidal fields. (vi) The basic error in the dynamo theory is discussed briefly. We point out that radical changes in dynamo theory have been suggested by Parker, but appear to have been ignored by others.
\end{abstract}

\section{Introduction}

In a series of papers (Piddington, 1971a, b, 1974, 1975a, d, e) I have developed a theory of solar magnetic fields which might be termed the primordial field theory. One of its important features is that submerged magnetic fields are in the form of helically twisted flux ropes as shown in Figure 1a, an idea proposed originally by Babcock (1961). A puzzling feature of Babcock's paper is that it is often quoted as though it also provided observational support for the dynamo theory (see, for example, Parker, 1970a; Stix, 1974) which is the very antithesis of the primordial field theory. Hence, before reviewing the latter it is desirable to explain this misunderstanding.

The dynamo theory describes two separate and distinct phenomena as follows.

(i) As pointed out some decades ago (see Elasser, 1956; Cowling, 1953), nonuniform rotation $\omega(\theta)$ of a conducting sphere will draw out a poloidal field to form a stronger, mainly toroidal field. This $\omega$-effect operates equally well with or without the added feature of twisted flux ropes and in Figure $1 \mathrm{~b}$ the result is illustrated with twists. There is no argument about this $\omega$-effect, which is common to both theories and is readily tailored to explain the butterfly diagram and the polarity of sunspot magnetic fields for an 11-yr cycle.

(ii) The second dynamo phenomenon is 'cyclonic turbulence' (Parker, 1955, 1970b) illustrated in Figure 1c. A rising convective cell carries some flux upwards and Coriolis forces rotate the flux loop to provide one of many elements of a reversed poloidal field. These elements are merged by turbulence to provide the $\alpha$-effect, whose very essence is dominance of the convective motions which create new 
(a)

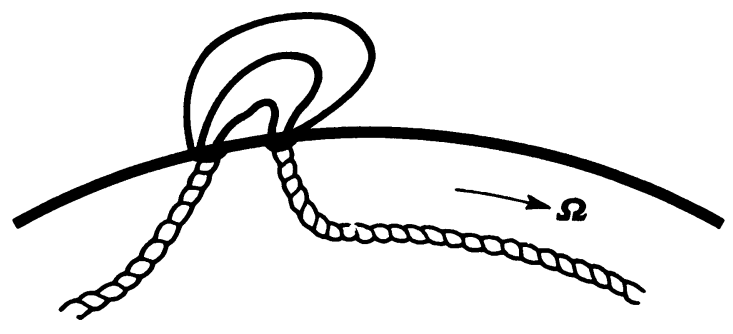

(b)

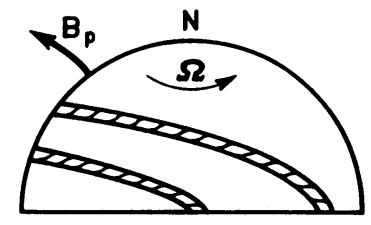

(c)

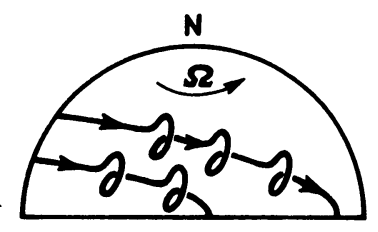

Fig. 1. Solar magnetic fields according to different theories. (a) A section of one of Babcock's flux ropes which has erupted to provide a sunspot pair. (b) Submerged flux ropes wound into a mainly toroidal field. (c) A more-or-less uniform toroidal field system erupts in numerous cyclonic loops to provide the $\alpha$-effect of the $\omega \alpha$ solar dynamo.

small-scale magnetic fields and then tangle and merge them to form new large-scale fields. The $\alpha$-effect and the flux-rope model are thus completely incompatible because the submerged flux ropes are powerful and cohesive and unaffected by convective motions.

The explanation of the misunderstanding is now clear. Babcock's flux ropes represent a form of the $\omega$-effect and he attempted to use them to provide an $\alpha$-effect as well. While $99 \%$ of the ropes left the Sun, the remaining $1 \%$ were retained to merge and form a new poloidal field. This part of his model is not acceptable (Piddington, 1972a), and if we allow $100 \%$ of the ropes to leave the Sun then the model is divorced from the dynamo theory and no longer supports that theory by its agreement with observations.

The success of Babcock's model of the $\omega$-effect in explaining several features of spot groups was widely acclaimed, and in Sections 2 and 3 we review the extension of this comparison with more recently discovered observational features. In Section 4 we then show how the flux rope model may be made part of a 22-yr cycle by replacing the $\alpha$-effect by a primordial field together with a meridional oscillation which causes a small change in the $\omega(r, \theta)$ pattern. Finally, for completion, we comment in Section 5 on the solar dynamo theory in its more recent and more divided versions.

\section{Sunspots}

Figure 1a shows how Babcock (1961) accounted for a pair of sunspots with powerful, twisted magnetic fields. This model was widely acclaimed, yet most recent reviews (see, for example, Meyer et al., 1974) state that "after the flux emerges through the photosphere it is concentrated by supergranule convection to form a sunspot". We 
show below that this view is incorrect; meanwhile we explain how the flux of a powerful, twisted rope is temporarily scattered and so appears to be controlled by gas motions.

In Figure 2a an inverted $U$ section of a rope is seen approaching the surface, the magnetic field being contained by a higher external gas pressure $p_{e}$ given by

$$
p_{e}=p_{i}+B^{2} / 8 \pi,
$$

where $p_{i}$ is the internal gas pressure and $B$ the field strength. At a critical level (depth $\approx 1000 \mathrm{~km}) p_{e}$ is too small to satisfy this equation and activity starts. The diameter of a substantial flux rope is $D \approx 10^{4} \mathrm{~km}$ while the scale height is only $\approx 300 \mathrm{~km}$. Thus, when a layer of the rope of thickness only $D / 30$ projects through the critical level it expands upwards while the remainder of the rope remains intact. This is the first arch filament, and as the rope continues to rise layer after layer erupt in their turns to provide an Arch Filament System (AFS) discussed further below.

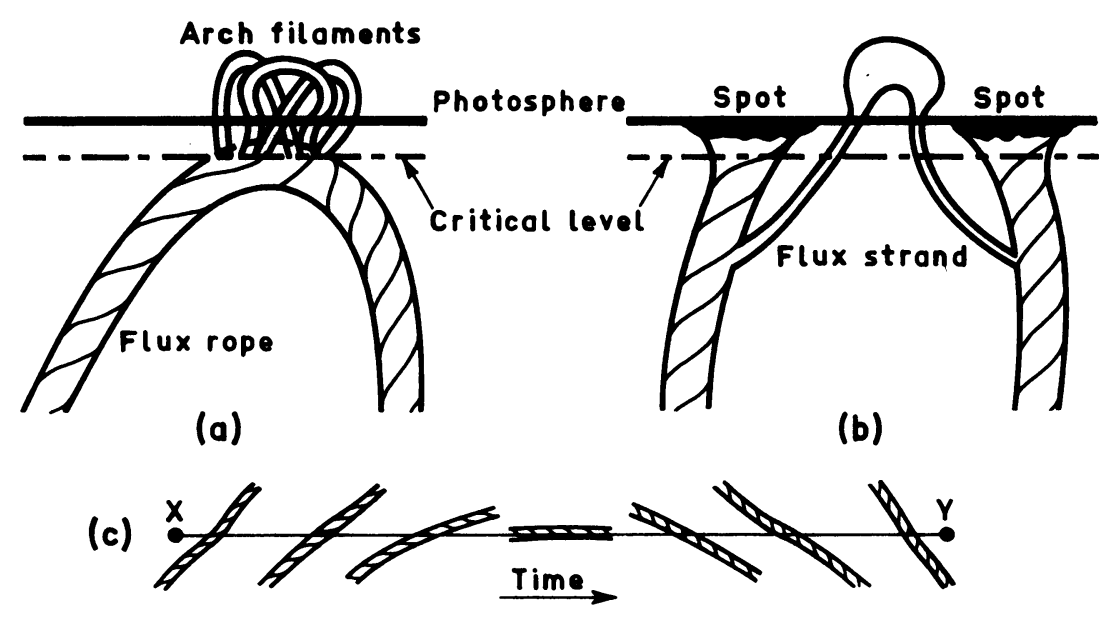

Fig. 2. Some details of the formation of an active region and spot pair. (a) A flux rope rises through the critical level and erupts layer by layer to provide a rotating Arch Filament System. (b) The two parts of the rope have separated and become nearly vertical. Some flux strands have unwound and separated from the rope. (c) A plan view of a steadily rotating Arch Filament System; rotation is caused by the twist in the rope.

Figure $2 b$ shows the two rope sections after the horizontal section has emerged, $a$ process which may take a couple of days corresponding to a velocity of $\approx$ $0.06 \mathrm{~km} \mathrm{~s}^{-1}$. A submerged flux rope is strongly cohesive and virtually indestructable, and we now see why scattered flux moves radially inwards towards a spot (Vrabec, 1974). It is because most flux remains connected to the submerged rope sections and is gathered together as those sections approach the surface. However, at this stage the ropes will start to unwind by the upward propagation of Alfvén waves; they will then 'fray' and lose some strands as shown in Figure $2 b$.

Returning to the AFS, we note that the model predicts a puzzling sequence illustrated in Figure 2c. The outer layer of a twisted rope will be strongly tilted from 
the axis of the rope whose projection on the surface is shown by the line $X Y$. Thus the first arch filament is tilted as shown by the left-hand filament. The tilt decreases to zero as the axis of the rope emerges and then increases in the opposite direction. Just such a remarkable sequence has been observed by Frazier (1972), to provide further confirmation that sunspot fields are indeed helically twisted. It will be seen that the effect may now be used to estimate the pitch of the helically twisted field.

In Paper I of this series we described several other features of a spot group which seem explicable only in terms of submerged helically twisted flux ropes. These include the marked asymmetry of spot groups, the ordered, large-scale proper motions of sunspots and the very long lives of some preceding (but not following) sunspots. For brevity, further discussion of these effects is omitted.

\subsection{The MAGnetic STRUCTURE OF A SUNSPOT}

Returning to the problem of the mode of creation of sunspots, we show in Figure 3 the left-hand section of an emerging flux rope. The horizontal part of the rope has been torn apart by internal pressure and the rope has partially untwisted and frayed into 'strands' which, as shown in the following section, are also helically twisted. Some of these cut the surface and so account for pores and smaller magnetic elements, while others marked $S$ are still submerged. The rope below the section $X X$ is intact and continues to move upwards as the rope section swings into a vertical position. It is easily seen that this upward movement must cause the pores and other surface magnetic elements to move radially inwards towards the small spot of central pore shown in Figure 3.

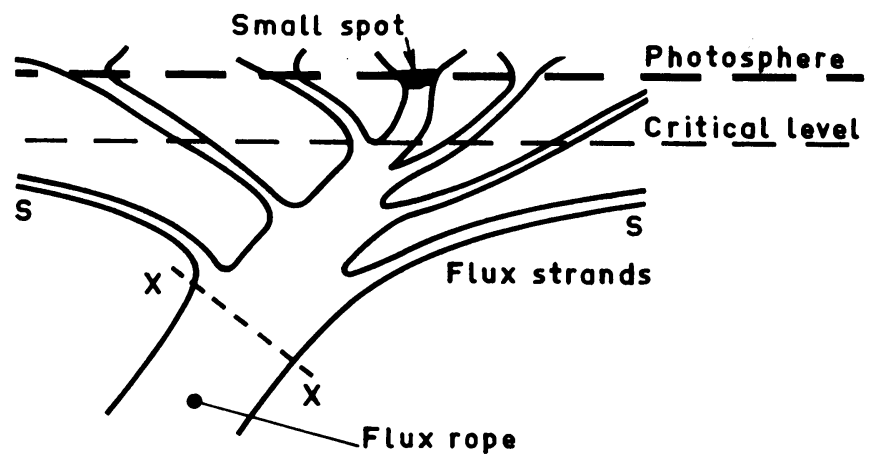

Fig. 3. A flux rope rising and gathering its frayed strands to provide a growing sunspot.

This process has been observed by Vrabec (1974) who also attributed it to magnetic control as in the model shown. The evidence for magnetic control (as opposed to the traditional idea of supergranule control) is as follows.

(i) Magnetic stresses make a rope strongly cohesive so that its submerged flux is not dispersed.

(ii) Over an area of more than 30 supergranule cells Vrabec (his Figure 2) observed ordered, convergent flow of magnetic features. Polarities of both signs are 
rather mixed yet only features of like polarity move towards the spot; adjacent features of opposite polarity do not move.

(iii) The paths of the moving magnetic features sometimes show marked curvature corresponding to the spiral structure of the spot penumbra. This effect is expected from the fact that the flux strands have partially unwound from the rope and so are curved.

(iv) The speeds of the magnetic features range up to $1 \mathrm{~km} \mathrm{~s}^{-1}$ which is $>2$ times that of supergranules. it is easily accounted for if some of the strands are nearly horizontal.

We have shown how flux accumulates above a rope end, and now consider why this causes pores and spots to be dark. It has been shown (Stenflo, 1973; Harvey and Hall, 1974 ) that even in quiet regions and even for magnetic elements of size $\approx 200 \mathrm{~km}$ the field strength is always $\approx 2000 \mathrm{G}$. On the other hand pores are known to form when the average field strength is only $\approx 1400 \mathrm{G}$. Accordingly (Paper IV) we suggest that pores are loosely twisted bundles of flux fibres whose total photospheric area is about $2 / 3$ that of the pore, the remaining $1 / 3$ being non-magnetic. The pores are dark because there is no convection within the individual strands, and also because convection between the strands is partly or wholly suppressed. One might ask why are the individual flux strands not observed as tiny pores, and the answer seems to be that they are. There are few really good white-light photographs, but Danielson's (1961, Figure 8 and others) Stratoscope I photos show pores which are so tiny that their lower limit cannot be distinguished from the dark intergranule regions.

According to this model a sunspot magnetic field has the form shown in Figure 4. It is made up of hundreds or thousands of flux strands whose flux may be typically $\approx 4 \times 10^{18} \mathrm{Mx}$ and field strength $\approx 4000 \mathrm{G}$ falling to $2000 \mathrm{G}$ at the photosphere

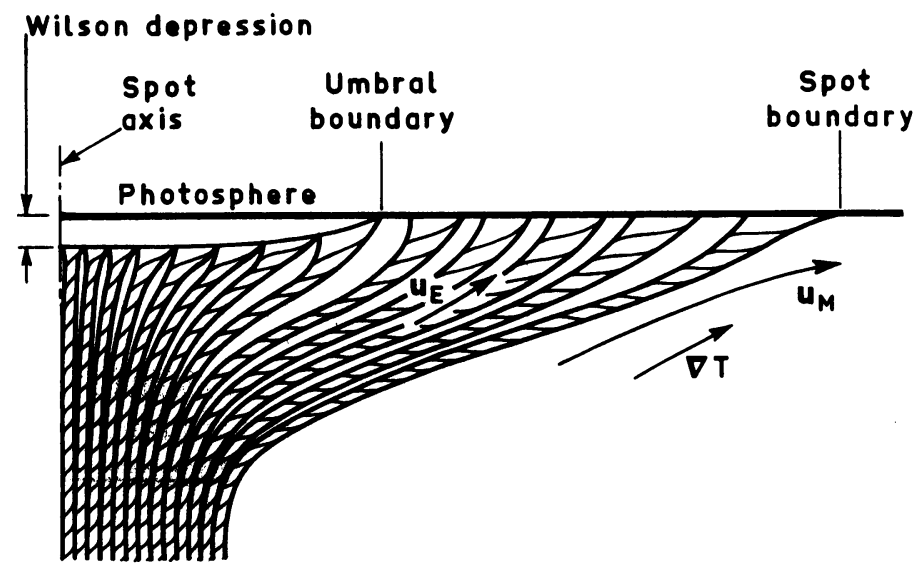

Fig. 4. A section of a sunspot magnetic field showing the individual twisted strands but, for simplicity, not showing the overall rope twist. The field in the subsurface strands is everywhere $\approx 4000 \mathrm{G}$. At the surface it is generally pressure limited to $\approx 2000 \mathrm{G}$ or less when the strand is tilted as in the penumbra. However, near the centre of a large spot the subsurface flux strands cannot expand and the full $4000 \mathrm{G}$ is seen at the surface. The outward Evershed flow $\mathbf{u}_{E}$ is a combination of flows of dark plasma within the flux strands and bright plasma between. The enhanced temperature gradient $\nabla T$ drives a new convective motion, the 'moat' convection $\mathbf{u}_{M}$, which accounts for the sunspot energy deficit. 
where it is pressure limited, except at the centres of large spots where its full value is observed. The twisted strands are twisted together (not shown) to form a rope with varying amounts of non-magnetic plasma between the fibres. Other features of the model are as follows.

(i) In the umbra the $2000-4000 \mathrm{G}$ strands completely fill the photosphere and we see only the colder magnetic plasma, thus accounting for the umbral darkness.

(ii) In the penumbra the dark magnetic plasma forms lanes between which the hot non-magnetic plasma convects. This is a modified granule convection in the form of long striae with characteristic period a few times the granule period. A similar convective pattern has been observed by Danielson and by Bray and Loughhead outside sunspots in regions between pores (see Paper IV).

(iii) The Wilson depression is explained in terms of completely isolated magnetic atmosphere described in Section 4 below. It is cooler than the main non-magnetic atmosphere, and at every level it adjusts its pressure according to Equation (1).

(iv) The rope model explains the origin of huge spots, some with diameters $\approx 10^{5} \mathrm{~km}$ (Newton, 1955) and some with central fields of $\approx 4000 \mathrm{G}$. It does not seem that these features could possibly be accounted for in terms of the compression of weak fields by the supergranule convective motions which are both too weak and of too small a scale.

\subsection{MOTIONS IN AND AROUND SUNSPOTS}

The fraying flux rope model of sunspot magnetic fields accounts for a number of plasma motions in and around spots. It explains, in the first place, why some spots vanish within a few days often accompanied by fragmentation while others last for weeks or months (Bumba, 1963). Spot fields without twists must disintegrate immediately by the flute instability, a fate which would overtake any spot formed in the traditional manner by supergranule motions. Twisted fields last as long as the twists and then decay as explained below. Other motions are as follows.

(i) In Paper I we discussed the proper motions of sunspots which are sometimes highly ordered over large distances and quite different for preceding and following spots. The motions are clearly the result of magnetic forces, and are not consistent with the traditional idea of supergranule control. They relate closely to the observed asymmetry of spot groups explained by Babcock (1961) in terms of flux ropes.

(ii) The model opens interesting possibilities for the explanation of light bridges, umbral granulation and umbral flashes. The submerged flux strands are separated by non-magnetic plasma which is not thermally isolated from the plasma outside the flux rope, and so is hotter than that in the strands. Upwellings of this plasma between the strands explain these curious effects.

(iii) In Paper IV we show how the Evershed outflow is related to the 'moat convection' and how each is explained. Partial suppression of convection beneath the 'umbrella' (Figure 4) will lead to an enhanced upward temperature gradient in the non-magnetic plasma and hence to a slow flow of hot gas $\mathbf{u}_{E}$ as shown. The gas within the flux tubes is cooler, thus accounting for the bright and dark curved striae of the penumbra; this gas provides an Evershed flow of cool gas within the individual, 
twisted flux strands. This flow is caused by the general upward movement of the whole rope system discussed above and in Section 4.

(iv) An extension of these ideas leads to simple explanations of the 'moat convection' and as yet unexplained sunspot energy deficit. Sheeley (1972) observed a new type of motion radially away from sunspots with velocities up to $1 \mathrm{~km} \mathrm{~s}^{-1}$ to distances of $\approx 2 \times 10^{4} \mathrm{~km}$, and Vrabec (1974) pointed out that this differs from supergranule motions in four major respects. We consider that this is a new non-random convective motion $\mathbf{u}_{M}$ (Figure 4 ) forced by the enhanced temperature gradient $\nabla T$ which develops beneath the penumbra.

In Paper IV we have only shown that this convective motion accounts readily for the sunspot energy deficit. A temperature difference (across the moat) of only $\approx 2 \%$ at a depth of $\approx 3000 \mathrm{~km}$ and a flow at speed $0.75 \mathrm{~km} \mathrm{~s}^{-1}$ carries away energy at a rate of $2 \times 10^{29} \mathrm{erg} \mathrm{s}^{-1}$ from a spot of radius $2 \times 10^{4} \mathrm{~km}$. This equals the energy deficit for such a spot, and the depth is appropriate because the thermal energy above that level is enough to provide the photospheric emission for $\approx 2$ days which seems a reasonable time scale.

(v) Finally we consider the motions which represent the slow decay of a sunspot (Harvey and Harvey, 1973; Vrabec, 1974). These moving magnetic features are isolated elements, which suggests that their submerged sections are twisted flux strands as depicted in Figure 5. They move independently, some overtaking others

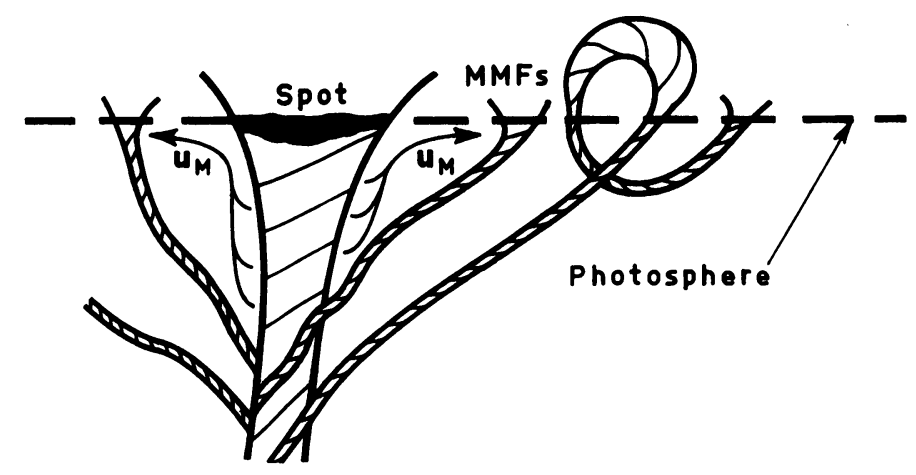

Fig. 5. A section of a flux rope below a decaying spot. Flux strands unwind to ever-increasing depths and are then carried across the moat as moving magnetic features (MMFs) by the moat convection $\mathbf{u}_{M}$. Strands are twisted and so the kink instability creates loops as shown.

and some moving along curved paths which match the penumbral filaments, which is explained, like the penumbral filaments, by flux strands which are unwinding from the flux rope. The total flux (the sum of the fluxes of both signs) transported is an order of magnitude more than the spot flux, but the net flux is just that of the spot. This effect is readily explained by the kink instability which will cause a succession of kinks to propagate up a flux strand as shown. The same effect accounts for the sudden appearance of features in the middle of the moat. Finally, we recall that long-lived spots decay by losing flux at a uniform rate, and explain the effect of the unwinding at a uniform rate of the outermost layer of flux strands. 
We suggest that these various phenomena in and around sunspots can only be explained in terms of the rope-strand model and confirms the features of the model inferred from earlier observations.

\section{Background Magnetic Fields}

After the spots have vanished, active regions expand and decay. The magnetic elements are clearly affected by the supergranule motions, but it is easily seen that this control is not complete as in the dynamo and other diffuse field theories. There are many reasons for this conclusion, one being the observations (Stenflo, 1973; Harvey and Hall, 1974) of magnetic elements of field strength $\approx 2000 \mathrm{G}$ and diameters only a few hundred kilometers. As shown in Paper IV these are only just explicable in terms of pressure limitation (Equation (1) above) in the low photosphere, and their preservation requires that the flux bundle concerned is twisted and so cohesive. Other evidence for a highly ordered subsurface magnetic structure is seen in the further development of the flux-rope-strand model as follows.

(i) A notable feature of the background magnetic fields is the unipolar magnetic region, which is a huge $\left(\widetilde{ }=10^{5} \mathrm{~km}\right)$ area with photospheric magnetic field of one strongly predominant polarity (see, for example, Livingston and Harvey, 1971, Figure 4). If solar magnetic fields could be manipulated by the convective eddies a very different picture would be seen, with the churned up fields having completely mixed polarities. The flux-rope model explains the result in terms of the structure shown in Figure 6, where the rope has untwisted down to the level $Z_{0}$. Above that level the rope has broken into smaller ropes and frayed into strands with flux typically $\approx 4 \times 10^{18} \mathrm{Mx}$, so that a rope of average flux $10^{21} \mathrm{Mx}$ has about 200 strands and large ropes up to $\widetilde{S} 10^{4}$ strands. From considerations of energy equipartition we have

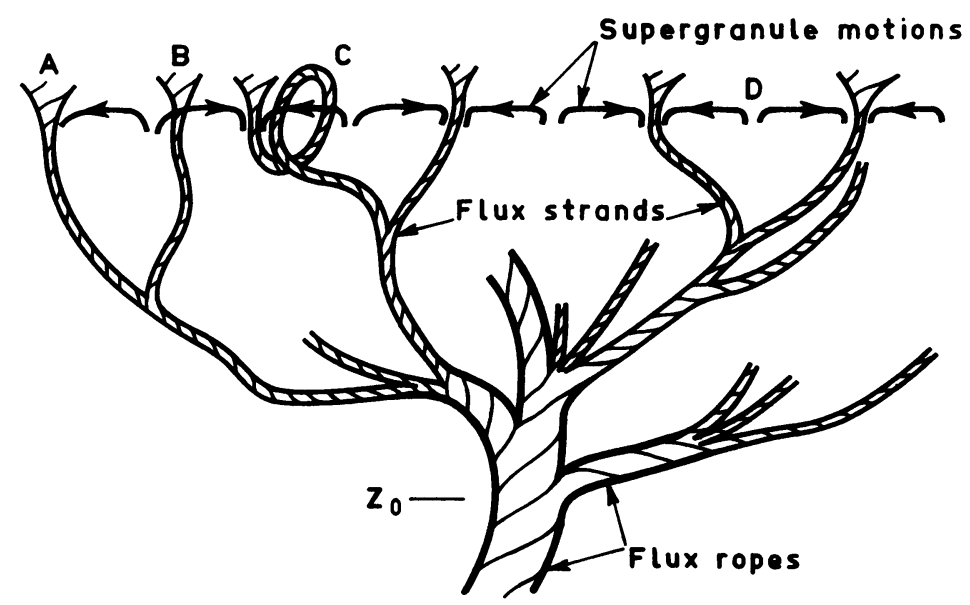

Fig. 6. A magnetic flux tree which is the structure beneath a unipolar magnetic region. The original rope (below the level $Z_{0}$ ) has split into smaller ropes and frayed into hundreds or even thousands of flux strands. Most of these are carried to the supergranule boundaries giving the illusion of control. Some are in the cell interiors, and some are looped. 
calculated that the strands may be bent to a radius roughly equal to that of a supergranule cell as shown. Only with this model does it seem possible to explain how the fields remain essentially vertical, yet are shuffled about by the supergranule motions and concentrated in the cell boundary regions (strand $A$, and others).

(ii) The model explains why some field concentrations are observed (Livingston and Harvey, 1975) in the interiors of cells as shown by strand $B$. This, and the fact that no weak fields are observed, shows that the flux cannot be concentrated by the supergranule motions as in the dynamo and similar diffuse-field theories.

(iii) Being helically twisted some strands will develop a loop (strand C) as a result of the kink instability, thus accounting for a small proportion of flux of opposite polarity in otherwise unipolar regions ('pepper and salt') and for tiny bipolar magnetic regions which appear and vanish (Frazier, 1970; Harvey et al., 1975). The loops also provide a likely explanation of the $\mathrm{X}$-ray bright points.

(iv) On occasions a supergranule cell may become surrounded by flux strands (cell D) whose total rigidity is then sufficient to control the convection. In this way we account for the observations (Livingston and Orrall, 1974) of cells with lifetimes up to 7 days.

\subsection{Migrations of MAgNetiC Regions}

Unipolar magnetic regions migrate over large distances and while proper motions are difficult to measure, Bumba and Howard (1965) have observed motions of about $0.1 \mathrm{~km} \mathrm{~s}^{-1}$. This figure is in reasonable agreement with that of Section 2 above of $0.06 \mathrm{~km} \mathrm{~s}^{-1}$ for the rate of upward motion of the ropes which must result in the 'peeling' of the ropes out of the Sun and their dissipation in space. In Paper V we have made a rough estimate of the rate of peeling for a rope of field strength $4000 \mathrm{G}$ lying at a depth of $10^{5} \mathrm{~km}$; the result is $0.13 \mathrm{~km} \mathrm{~s}^{-1}$. Thus observational and theoretical estimates lead to a figure of about $0.1 \mathrm{~km} \mathrm{~s}^{-1}$ or about 0.7 solar circumference per year.

This result explains a number of puzzling phenomena which do not agree with Leighton's (1964) model of dispersion of magnetic fields by a combination of differential rotation and random walk by supergranule motions. These phenomena are now explained in terms of the peeling of flux ropes having the configuration shown in Figure 7a.

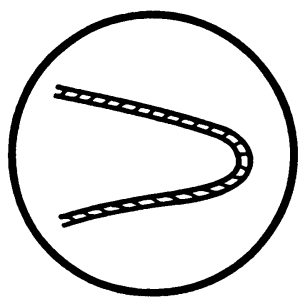

(a)

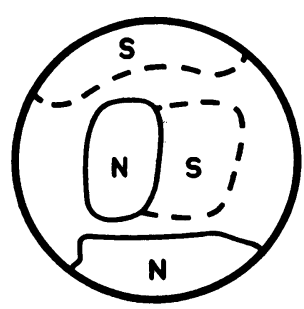

(b)

Fig. 7. Large-scale solar magnetic fields. (a) A submerged flux rope. (b) Surface background magnetic fields, illustrating the accumulation of $f$-region flux near the poles, and 'magnetic latitudes'. 
(i) Following $(f)$ magnetic regions migrate eastwards and polewards to cause flux accumulations at high latitudes (Figure 7b) and apparent reversal of a general field which, as seen in Section 4 , is probably only remnants of $f$-region flux. Babcock (1961) estimated that this flux would provide polar fields 100 times stronger than observed, and so there must be a dissipative mechanism. This was confirmed by Bumba and Howard (1965) who observed that most of migrating fields simply disappear by a process of expansion and weakening.

This effect is not explicable by the dynamo mechanism because huge areas of a single polarity simply fade away. We suggest that it is due to the peeling of a flux rope out of the Sun and its entire loss, as described in Section 4.

(ii) The synodic period of 27 days for recurrent geomagnetic storms and lower latitude solar background fields (see Newkirk, 1971) seems to indicate that the Sun has a rigidly rotating core which somehow controls surface features. However, the flux-rope model provides a more plausible interpretation of such uniform rotation.

The preceding $(p)$ magnetic regions migrate westwards and equatorwards with speeds of $\approx 0.1 \mathrm{~km} \mathrm{~s}^{-1}$ and along paths as delineated by the flux rope of Figure $7 \mathrm{a}$. The $p$ regions which originate at latitudes near $\pm 40^{\circ}$ move mainly westward, and by adding their proper motion to the rotational surface speed of $\approx 1.3 \mathrm{~km} \mathrm{~s}^{-1}$ the synodic period is reduced from 29 days to $\approx 27$ days. Near the equator the proper motion is equatorwards and so does not change the synodic period which is 27 days, so that there is a strong tendency for a general rotation at this period.

(iii) The remarkable feature of active longitudes, or the appearance of new active regions within or close to old $p$ regions, is explained by the flux-rope model. As a $p$ rope section is peeled from the Sun it disturbs an adjacent rope and causes it to erupt; the ropes may have some magnetic connection, or the effect may be due to turbulence. Since $f$ regions migrate polewards they traverse latitudes which were active earlier in the 11-yr cycle and so have lost their ropes; thus active longitudes tend to involve $p$ rather than $f$ regions.

(iv) Finally, there is the puzzling phenomenon of magnetic longitudes which stretch across the equator as shown in Figure 7b (see Stenflo, 1972; Svalgaard et al., 1974). This is simply an extension of active longitudes, for the case where the flux rope is peeled out across the equator so that the $p$ magnetic regions invade the opposite hemisphere. It is easily seen that if two active longitudes develop on opposite sides (front and back) of the Sun and in opposite hemispheres they will turn the Sun into a dipole with its axis in the equatorial plane as sometimes observed (Wilcox, 1971).

It may now be seen that the combination of polar fields and magnetic longitudes shown in Figure $7 \mathrm{~b}$ is just that invoked by Svalgaard et al. (1974) to explain large-scale coronal and interplanetary magnetic fields.

\subsection{THE PHOTOSPHERIC AND CHROMOSPHERIC NETWORKS}

Let us turn from the large-scale magnetic features to those which are known to be responsible for the facular network. The structural features of the network have been reviewed recently by Bray and Loughhead (1974) and Michard (1974), and the main ones are shown in Figure $8 \mathrm{a}$ which follows earlier sketches of Beckers and of 


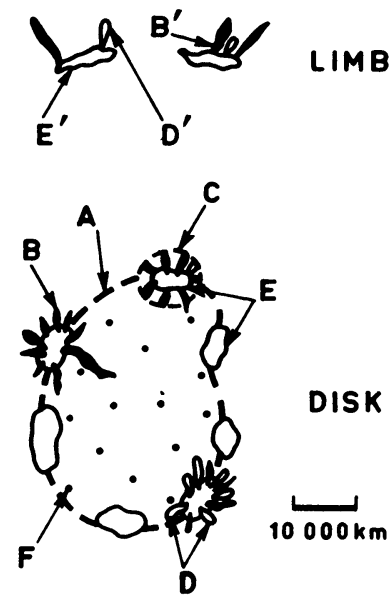

(a)

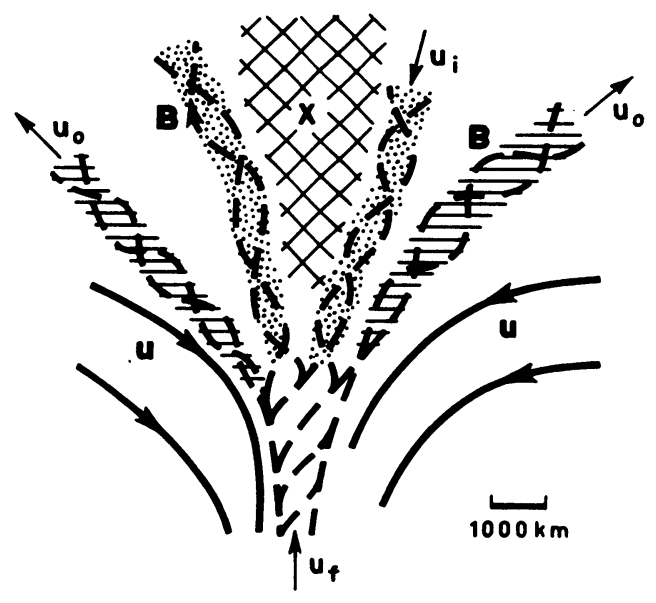

(b)

Fig. 8. The chromospheric network. (a) The various network elements comprising dark and bright mottles, rosettes and bushes, spicules and grains (after Beckers). (b) Their structure is explained in terms of a flux strand which frays in the chromosphere into $\approx 10$ flux fibres.

Michard. We explain these various elements in terms of the individual flux strands shown in our earlier Figure 6, and now shown in further upward projection in Figure $8 \mathrm{~b}$. The base of this strand is in or below the photosphere, and the strand has untwisted and frayed into a dozen or so flux 'fibres'. Since a typical strand has a flux of $\approx 4 \times 10^{18} \mathrm{Mx}$, a typical fibre has a flux of $\approx 4 \times 10^{17} \mathrm{Mx}$. The models of the various elements are as follows.

(i) Around the boundary of a supergranule cell $A$ are distributed about ten network elements each attributed to a single flux strand. The various fibres of each strand delineate the dark mottles $B$, a group of which may form a rosette $C$. Another common network element is the chain (or double chain), which is not shown but is explained as a particularly loose bundle of fibres which has been compressed and flattened by supergranule motions.

(ii) On the limb rosettes become bushes and it seems likely that dark mottles appear as spicules $B^{\prime}$ which are intermittent emissions of plasma $\left(u_{0}\right)$ caused by the buffeting of a flux fibre by the granule motion u (Piddington, 1972b). The twisted fibres account for the observed rotation of spicules and for the fact that they are long and slender. Some ejected plasma will fall back and some may traverse the corona and fall into a distant flux strand $\left(u_{i}\right)$.

(iii) As well as dark mottles there are bright mottles $D, D^{\prime}$ which form a structure $E, E^{\prime}$ within and below the rosette or bush. These elements might be attributed to hot non-magnetic gas ( $X$ in Figure $8 b$ ), heated by transverse oscillations of the flux fibres and perhaps identical with Giovanelli's (1974, Figure 5) 'diffuse' component. The vibrations of the flux fibres (Alfvén waves) are, in turn, attributed to granule motions at lower levels.

This rather tentative magnetic model of the photospheric and chromospheric network gives some idea of the great difficulties which will be met in attempting a 
detailed plasma model. One-or two-component models seem unlikely to satisfactorily explain the main features which include hot and cold non-magnetic plasma and inward and outward moving magnetic plasma.

(iv) As seen in Figure 6, every flux strand is connected to its flux rope to form a tree-like structure, with the outer strands all tending to be tilted away from the axis of the tree trunk. This might explain the ordered 'porcupine' structure of some fields of spicules (Bray and Loughhead, 1974, p. 37). Again, as the whole magnetic structure or 'tree' is migrating, one might expect to see the upper branches of the tree all tilted away from the direction of migration. In this way we explain the puzzling 'wheat field' pattern of spicules.

(v) In the interior of the cells we see the fibrils, some of which may be unusually long dark mottles. Some, however, which occur in active regions are a different phenomenon, caused by a flux fibre which loops across one or two supergranule cells rather than rising into the corona as do the great majority of flux fibres.

(vi) Also in the cell interior are the dark and bright grains ( $F$ in Figure 8b) which are attributed to the stray magnetic elements known to occur within the cells (Livingston and Harvey, 1975). The grains are then smaller examples of the network elements $C$ and $\mathrm{E}$.

\section{The Poloidal and Toroidal Field Systems}

The primordial field, or flux-rope, model of solar magnetic fields is based firmly on the observations of surface fields described by Babcock (1961) and greatly extended in later papers summarized above. All of these effects are caused by toroidal fields, and so we require an explanation of the origin of these toroidal fields and the reason why they reverse every 11 years. We have suggested earlier (Piddington, 1971a) that they are wound from a non-reversing and presumably primordial poloidal field. The observational evidence for the existence of such a field is discussed in Paper IX (in preparation) of this series and is summarized briefly here.

(i) The observed reversals of the polar fields during 1957-58 (Babcock, 1959) were taken as proof of the reversal of a 'general' poloidal field, an effect which only seems explicable in terms of a dynamo field. Today the situation has changed drastically, and it is known that the highly erratic behaviour of the polar fields is caused primarily by the migration to those regions of a large amount of flux of the $f$ magnetic regions. There is no evidence that these fields have merged with $\alpha$-effect (dynamo) fields at lower latitudes to provide a 'general' poloidal field. On the other hand it is quite possible that the f-region fields are so strong that they usually hide a weaker primordial field. A choice between these possibilities must be made on the basis of other evidence.

(ii) Bumba et al. (1968) report the observation of magnetic fields of a new cycle forming in one broad longitude zone, in an area where weak fields from the old cycle were still visible. This result is explicable only in terms of new toroidal fields which are wound below the old, presumably from a permanent poloidal field.

(iii) It is reasonably well established that solar magnetic fields are often delineated by plasma structures, and during the eclipse of 1954 , June 30 the coronal structure 
revealed an almost flawless dipole field. Waldmeier (1955) termed this a new, undisturbed form as distinct from the so-called minimum form which always reveals relics of active region fields. We suggest that on this unique occasion we were allowed a glimpse of a true, deep-seated dipole-type field, the polarity being positive or outwards in the northern hemisphere.

The alternative explanation, that the field is a dynamo field, is most unlikely because such a field is patched together from cyclonic loops which, observationally, are identified with the fields of active regions. These and their remnants are always highly irregular in structure, and a highly ordered and symmetric field seems possible only when the background fields have left the Sun entirely as required in the primordial field model.

(iv) It has often been suggested that a primordial field would have been lost during the Hayashi phase of turbulence. This argument is based on the concept of turbulent or eddy diffusion which has been shown in Papers II and III to be false.

\subsection{THE TOROIDAL FIELD SYSTEM}

All important theories of solar magnetic fields agree on one process: that a toroidal field arises from the progressive winding up of a poloidal field by the latitude variation $\partial \omega / \partial \theta$ of the non-uniform rotation. They also agree that after attaining a certain stage of development the submerged fields will erupt to provide active regions and spot groups. By adjusting the various parameters it is then a simple matter to account for the observed butterfly diagram, so that this feature is common to all theories and provides no particular support for the dynamo theory.

A major addition to these ideas was Babcock's (1961) concept of rolling the toroidal flux into flux ropes whose cohesive forces made them immune to convective motions. Such twists account for so many observational features that they must be an accepted feature of solar fields. However, twisted flux ropes cannot provide the dynamo $\alpha$-effect and so must be divorced from dynamo theory. The subsurface field system inferred from observations is now totally different from that of the dynamo fields, which comprise a generally diffuse and tangled field system which is dominated by the convective motions to provide the $\alpha$-effect.

Submerged flux ropes have field strength of $\approx 4000 \mathrm{G}$, and if the surface fields have a strength averaged over the whole sum of, say, $20 \mathrm{G}$ then the vertical ropes occupy only $\approx \frac{1}{2} \%$ of the convection zone. The remaining $99 \frac{1}{2} \%$ is non-magnetic and the two plasmas provide two entirely separate and mainly isolated atmospheres. The isolation is imposed, of course, by the very large electrical conductivity in the convection zone (Paper II) and the consequent very slow rate of diffusion of plasma across field lines. The isolation allows the plasma pressure inside the flux-rope system to adjust itself to satisfy Equation (1) at all levels. This is not possible if strong fields are formed in the traditional manner by the compression of weak fields.

The next problem is that of the elimination of the flux ropes of one 11-yr cycle before, or during, their replacement by those of the following cycle. We suggest that all toroidal fields must leave the Sun and float away, and most may be lost simply by the peeling process discussed in Section 3. If this process continues until the ropes are peeled back to their junctions with the poloidal field, then most of the plasma within 
the flux ropes flows back into the shearing regions where it existed before the ropes were created.

It is possible, however, that some ropes are broken in two or more places and that we must account for the disposal of the large amount of plasma within these rope sections. Such a section will become shorter and shorter as it is peeled from both ends, but its plasma cannot escape. However, the section must eventually lose all of its twists and fragment into fibres of diameter $<100 \mathrm{~km}$. In a thin layer within the photosphere the electrical conductivity falls to a sufficiently low value for these fibres to be separated from their plasma within a few days and so they are also lost from the Sun.

\subsection{REVERSAL OF THE TOROIDAL FIELDS}

If the Sun does have a significant primordial field then, somehow or other, the field and the differentially rotating plasma must have interacted and evolved so that, averaged over long periods, the magnetic field lines lie on surfaces of isorotation. This does not mean that at all times they lie on those surfaces, otherwise there would be no $\omega$-effect and no toroidal fields at all. For 11 years the field lines of a poloidal field must tilt one way relative to the $\omega=$ const. surfaces, and for the next 11 years they must tilt in the opposite way. A simple form of such a model is shown in Figure 9, which was inferred from observations of meridional oscillations by Richardson and Schwarzschild (1953), and also deduced as an essential alternative to the untenable $\alpha$-effect (Piddington, 1971a).

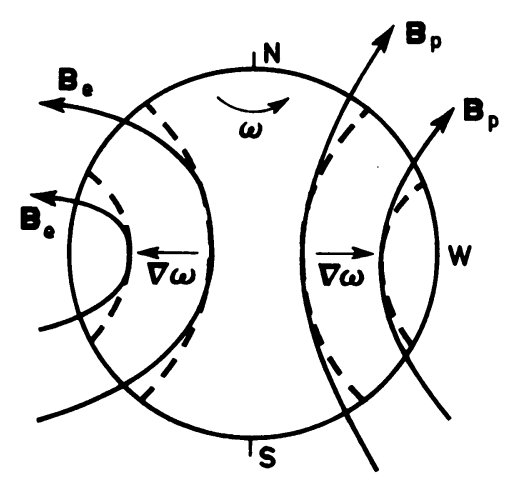

(a)

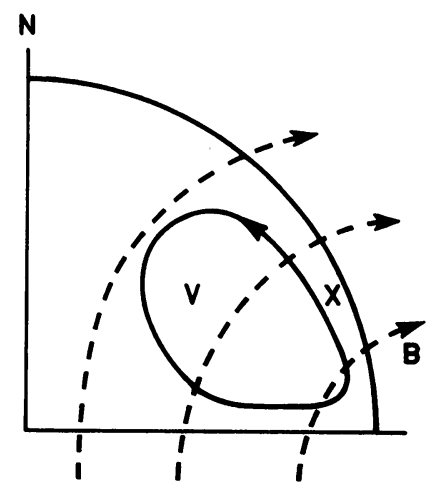

(b)

Fig. 9. Illustrating the mechanism of reversal of the solar toroidal magnetic fields. (a) A dipole-like magnetic field has its lines of force lying, on an average, on the surfaces of isorotation; for 11 years they are tilted equatorwards of these surfaces $\left(B_{e}\right)$ and for 11 years polewards $\left(B_{p}\right)$. Consequently two opposed sets of toroidal fields are created by the shears $\nabla \omega$. (b) A meridional oscillation which could change the field $B$ from the equatorial form $B_{e}$ to the polar form $B_{p}$.

Figure 9a shows a solar polar section with hypothetical surfaces of rotation as dashed lines. It will be seen that $\nabla \cdot \omega$ is outwards which is a requirement needed to account for the twists observed in sunspot magnetic fields (Babcock, 1961), and is, incidentally, the opposite to that required by dynamo theory (Stix, 1974). The figure 
also shows two halves of a permanent poloidal field as they appear during the two halves of a 22-yr cycle. On the left is the 'equatorial' form $B_{e}$, with the emerging field lines tilted equatorwards of the isorotation surfaces. On the right is the 'polar' form $B_{p}$ with the opposite direction of tilt. In Figure $9 \mathrm{~b}$ is shown a single quadrant of a meridional oscillation $V$ which, with speeds of only $\approx 100 \mathrm{~cm} \mathrm{~s}^{-1}$, will transform the $B_{e}$ field to the $B_{p}$ form. The toroidal fields wound by the $\omega$-effect from $B_{p}$ is opposite that wound from $B_{e}$; each successive set of toroidal ropes is wound below its predecessor and helps to push the latter out of the Sun. Thus, at least kinematically we have explained the solar 22-yr cycle in terms of a primordial field and a meridional oscillation.

While the dynamo theory is limited to kinematics, we have been able to provide a simple dynamical picture of the above hydromagnetic oscillator (Piddington, 1971b). In the region $X$ of Figure $9 \mathrm{~b}$ the magnetic field is sheared and so resists and eventually reverses the motion to provide a simple harmonic oscillator. Reasonable values of the various parameters lead to a period of $18 \mathrm{yr}$ in good agreement with that observed. There is even the possibility of explaining the excitation of the meridional oscillation in terms of meridional forces exerted by the toroidal fields.

There is an interesting alternative to the simple model of Figure 9, which requires no change in the poloidal field. Instead of the above $V$ oscillation we invoke an oscillation which involves an ordered transfer of angular momentum $\omega(r, \theta)$. In this way the surfaces of isorotation oscillate back and forth across the stationary field lines. The study of these different hydromagnetic oscillators should provide interesting problems and perhaps a true picture of solar magnetic fields.

\section{Discussion of the dynamo Theory}

It has often been stated that the "main features of the dynamo that produces the solar cycle are now generally accepted" (Weiss, 1971). Yet as shown in Papers I, IV and V and in the above review most observational evidence is opposed to a dynamo theory and theoretical arguments given in Papers II and III appear to raise fatal difficulties. We believe that the sequence of events leading to this disagreement may be summarized as follows.

(i) Cosmic dynamo theory opens fascinating possibilities, and the winding of a toroidal field ( $\omega$-effect) provided a major piece of the mechanism which Parker (1955) completed with his cyclonic turbulence or $\alpha$-effect. However, apart from the solar differential rotation, the model lacked observational support until Babcock's (1961) model appeared to provide such support. Overlooked was the fact that Babcock's model is concerned mainly with the $\omega$-effect, which is not in dispute, and his attempt to reverse the poloidal field was neither necessary nor satisfactory (Section 4 above). The flux-rope concept may be adopted without difficulty by the primordial field model, thereby removing the only major flaw in Babcock's model.

(ii) The next major event in this sequence was the discovery of the supergranulation and of its apparent control of the magnetic fields. At this stage the concepts of cyclonic turbulence, flux ropes and supergranule control were taken as complementary and as providing a sound observational basis for dynamo theory. In fact, the 
reverse is true: as seen in Sections 2 and 3 flux ropes evolve almost entirely under the influence of magnetic stresses and are quite incompatible with the $\alpha$-effect.

(iii) In turning to the theoretical side, we must again go back two decades to Elsasser's (1956) assumption that just as turbulence disperses smoke, heat, or any other passive property of a fluid, so it will eliminate a passive magnetic field. This assumption has gained general recognition and it is difficult to overestimate its pervasive influence in hydromagnetic turbulence theory.

The relevant equation for the magnetic field $\mathbf{B}$ is

$$
\frac{\partial \mathbf{B}}{\partial t}=\nabla(\mathbf{V} \times \mathbf{B})+\eta \nabla^{2} \mathbf{B},
$$

where $\mathbf{V}$ is the plasma velocity and $\eta$ the diffusivity. The point is that turbulence $V_{t}$ is part of the $V$ term and represents transport of the field lines with the plasma, while only the $\eta$ term represents transport through the plasma. There are two similar terms in the case of a scalar quantity (Paper III) but here the physical results of both are much the same, leading to Elsasser's error. Using $V_{t}$ as a diffusion effect leads to a new diffusivity (Leighton, 1964; Rädler, 1968; Parker, 1970a, 1971; Vainshtein and Zel'dovich, 1972; Nakagawa and Priest, 1973; Stix, 1974; Meyer et al., 1974)

$$
\eta_{t} \approx 0.2 V_{t} L,
$$

where $L$ is the characteristic eddy dimension. If we now insert $\eta_{t}$ in place of $\eta$ in Equation (2) we increase the diffusion rate in the convection zone by a factor of $\widetilde{ } 1^{7}$. The electrical resistivity is supposed to be similarly increased.

The above procedure is an essential part of modern dynamo theory, yet it is quite unjustified (Piddington, 1972a, 1973, Paper III; Parker, 1973a) and invalidates solar dynamo theory.

(iv) The above procedure not only grossly overestimates the diffusivity, but hides an even more serious error in dynamo theory. By taking $V_{t}$ away from the $\mathrm{V}$ term of Equation (2) we lose sight of the fact that these motions cause rapid amplification of the magnetic fields by shear, so that instead of eliminating unwanted fields they build them up. Parker (1973a, b) has attempted to meet this difficulty by two drastic revisions of the dynamo theory. First, instead of the magnetic loops formed by cyclonic turbulence merging rapidly, the fields are drawn out into long, thin filaments. When a filament is thin enough it is able to merge with an oppositely directed neighbour and vanish. Second, in order to accelerate merging over the rate allowed by molecular diffusion, the Petschek mechanism is invoked.

This new dynamo shows even less agreement with observations and, in our opinion, less plausibility than the old. Additionally, it has been analysed (Paper III, Section 4) and appears to fail on theoretical grounds; fields either halt convection or become chaotic.

(v) We have seen above the diffusivity used in the dynamo is far too large, that turbulence creates new fields faster than it destroys them, and that Parker has, accordingly, proposed a radically new 'thin filament' dynamo. Yet in a recent review, Stix (1974) has virtually ignored these developments. Without giving any physical arguments he repeats the claim that transport of magnetic field with the gas is equivalent to diffusion through the gas. 


\section{References}

Babcock, H. D.: 1959, Astrophys. J. 130, 364.

Babcock, H. D.: 1961, Astrophys. J. 133, 572.

Bray, R. J. and Loughhead, R. E.: 1974, The Solar Chromosphere, Chapman and Hall, London.

Bumba, V.: 1963, Bull. Astron. Inst. Czech. 14, 91.

Bumba, V. and Howard, R.: 1965, Astrophys. J. 141, 1502.

Bumba, V., Howard, R., Martres, M. J., and Soru-Iscovici, I.: 1968, in R. Howard (ed.), 'Structure and Development of Solar Active Regions', IAU Symp. 35, 13.

Cowling, T. G.: 1853, in G. P. Kuiper (ed.), The Sun, University Chicago Press, p. 532.

Danielson, R. E.: 1961, Astrophys. J. 134, 312.

Elsasser, W. M.: 1956, Rev. Mod. Phys. 28, 135.

Frazier, E. N.: 1970, Solar Phys. 14, 89.

Frazier, E. N.: 1972, Solar Phys. 26, 130.

Giovanelli, R. G.: 1974, Solar Phys. 38, 117.

Harvey, J. and Hall, D.: 1974, Bull. American Astron. Soc. 6, 81.

Harvey, J. and Harvey, K.: 1973, Solar Phys. 23, 61.

Harvey, K. L., Harvey, J. W., and Martin, S. F.: 1975, Solar Phys. 40, 87.

Krause, F. and Rädler, K.-H.: 1971, in R. Howard (ed.), 'Solar Magnetic Fields', IAU Symp. $43,770$.

Leighton, R. B.: 1964, Astrophys. J. 140, 1547.

Leighton, R. B.: 1969, Astrophys. J. 156, 1.

Livingston, W. and Harvey, J.: 1971, in R. Howard (ed.), 'Solar Magnetic Fields', IAU Symp. 43, 51.

Livingston, W. and Harvey, J.: 1975, American Astron. Soc., Solar Phys. Div., Meeting Boulder, Colorado.

Livingston, W. C. and Orrall, F. Q.: 1974, Solar Phys. 39, 301.

Meyer, F., Schmidt, H. U., Weiss, N. O., and Wilson, P. R.: 1974, Monthly Notices Roy. Astron. Soc. 169 , 35.

Michard, R.: 1974, in R. G. Athay (ed.), 'Chromospheric Fine Structure', IAU Symp. 56, 3.

Nakagawa, Y. and Priest, E. R.: 1973, Astrophys. J. 179, 949.

Newkirk, G.: in R. Howard (ed.), 'Solar Magnetic Fields', IAU Symp. 43, 547.

Newton, H. W.: 1955, Vistas Astron. 1, 666.

Parker, E. N.: 1955, Astrophys. J. 122, 293.

Parker, E. N.: 1970a, Ann. Rev. Astron. Astrophys. 8, 1.

Parker, E. N.: 1970b, Astrophys. J. 162, 665.

Parker, E. N.: 1971, Astrophys. J. 163, 279 and 164, 491.

Parker, E. N.: 1973a, Astrophys. Space Sci. 22, 279.

Parker, E. N.: 1973b, Astrophys. J. 180, 247.

Piddington, J. H.: 1971a, Proc. Astron. Soc. Australia 2, 7.

Piddington, J. H.: 1971b, Solar Phys. 21, 4.

Piddington, J. H.: 1972a, Solar Phys. 22, 3.

Piddington, J. H.: 1972b, Solar Phys. 27, 402.

Piddington, J. H.: 1973, Astrophys. Space Sci. 24, 259.

Piddington, J. H.: 1974a, in R. G. Athay (ed.), 'Chromospheric Fine Structure', IAU Symp. 56, 269.

Piddington, J. H.: 1975a, Astrophys. Space Sci. 34, 347, Paper I.

Piddington, J. H.: 1975b, Astrophys. Space Sci. Paper II 35, 269.

Piddington, J. H.: 1975c, Astrophys. Space Sci. Paper III 38, 157.

Piddington, J. H.: 1975d, Astrophys. Space Sci. Paper IV.

Piddington, J. H.: 1975e, Astrophys. Space Sci. Paper V.

Rädler, K. H.: 1968, Z. Naturforsch 23a, 1851.

Richardson, R. S. and Schwarzschild, M.: 1953, Academia Lincei, Convegno 11, Rome 1952, p. 228.

Sheeley, N. R.: 1972, Solar Phys. 25, 98.

Simon, G. W. and Leighton, R. B.: 1964, Astrophys. J. 140, 1120.

Stenflo, J. O.: 1972, Solar Phys. 23, 307.

Stenflo, J. O.: 1973, Solar Phys. 32, 41.

Stix, M.: 1974, Astron. Astrophys. 37, 121.

Svalgaard, L., Wilcox, J. M., and Duvall, T. L.: 1974, Solar Phys. 37, 157.

Vainshtein, S. I. and Zel'dovich, Ya. B.: 1972, Usp. Fiz. Nauk. 106, 431; Soviet Phys. Usp. $15,159$.

Vrabec, D.: 1974, in R. G. Athay (ed.), 'Chromospheric Fine Structure', IAU Symp. 56, 201.

Waldmeier, M.: 1955, Z. Astrophys. 36, 275.

Weiss, N. O.: 1971, in R. Howard (ed.), 'Solar Magnetic Fields', IAU Symp. 43, 757.

Wilcox, J. M.: 1971, Publ. Astron. Soc. Pacific 83, 516. 


\section{DISCUSSION}

Stenflo: Can you explain how the twisting of the toroidal magnetic flux is produced in the first place?

Newkirk: What supplies the energy to twist the ropes in Piddington's theory?

Giovanelli: I think that it is differential rotation, which Piddington does not attempt to explain but takes as an observed fact. You will recall that Babcock, whom Piddington follows, also invoked twisting due to buffeting or shear by convective eddies.

Parker: I would like to point out the origin of Piddington's divergent view, the 'Piddington heresy', in contrast to the orthodox dogma that the solar magnetic fields are the result of a hydromagnetic dynamo. The basis is that Piddington does not believe in turbulent diffusion. Without turbulent diffusion the magnetic lines of force are locked into the solar gas because of the high electrical conductivity. The resistive decay times are $10^{5}$ yr or more, instead of $10 \mathrm{yr}$. Magnetic lines of force do not reconnect, and the hydromagnetic dynamo cannot operate. Piddington's disbelief in turbulent diffusion is based on the fact that the turbulent eddies wrap and stretch the magnetic field into filaments, with the field strength in each filament growing exponentially in time. Thus the mean square field quickly reaches the equipartition value and halts the turbulence. Piddington argues that turbulent diffusion of the mean fields does not proceed beyond this point.

Piddington has put his finger on a weak spot in turbulence theory. There is no formal deductive answer to his objection to turbulent diffusion. All 'theories' of turbulent diffusion of the mean field merely take it for granted that there is no problem with the growth of the mean square field. Yet the elementary calculation shows the exponential growth.

The answer to Piddington's objection to turbulent diffusion is, I think, the rapid reconnection of the lines of force of neighboring strands of field. This reconnection takes place at a fraction of the Alfvén speed (as pointed out by Priest this morning). The strands are continuously cut to pieces and the exponential growth does not occur. Thus I believe that the turbulent decay of a weak mean field goes on relatively unhindered by the tensions in the individual small-scale strands. Indeed, on the basis of a simple statistical hypothesis, it is possible to show that the mean magnetic field diffuses in the same way as a scalar field (such as smoke) carried in the same fluid.

Piddington has developed his ideas of the behaviour of magnetic flux in the Sun in response to his view that it is permanently frozen into the fluid.

If I may comment now on one aspect of Piddington's picture of the magnetic tree whose emergence carries the active region to the surface, it is to note that an essential part of his idea is the unwrapping of the twisted flux rope at its apex where it emerges through the surface. It is a theoretical fact that magnetic buoyancy pushes the twisted coils upward along this tube, concentrating them in the apex. Thus, the apex is the place of maximum twisting, rather than the place of least twisting suggested by Piddington. I do not understand how the twisted rope is to fray, coming at its apex to form the many separate features of the active region.

Giovanelli: The tube expands greatly when it reaches the outer solar layers where the external gas pressure is too low to confine the magnetic field. The twisting is unable to prevent this expansion. There would then be a discontinuity at the surface, which is eliminated by the upward propagation of the twists from just under the surface, the process which Piddington associates with 'fraying' in the case of a rope consisting of many strands.

A participant: I should expect that the ropes would simply untwist completely with the Alfvén velocity as soon as they break through the photosphere and that the flux concentration dilemma still remains.

Priest: The sunspot field which you assume is, in my view, unlikely. If one forms a flux tube by bringing together many twisted fibres, rapid reconnections will occur, leaving a simply twisted field. It is easy to reconnect field lines which are aligned arbitrarily and not just at $180^{\circ}$.

Further the amount of twist in your diagrams is greatly exaggerated. Raadu showed that, as soon as field lines are twisted more than once around the axis of a finite tube, the field becomes unstable to the kink instability. Can the observers tell us how common are spiral penumbral structures?

Giovanelli: In white light the penumbral fibrils are fairly straight and radial, but at times fibrils seem to cross at angles, contrary to what Dr Priest has suggested (but there may be a vertical separation between them). The superpenumbral fibrils seen in $\mathrm{H} \alpha$ are much longer, and many show curved spiral structure.

Stenflo: Hale noticed the $\mathrm{H} \alpha$ vortex pattern around sunspots already in 1904. Later statistical analysis by Richardson showed that a large fraction of all sunspots have a clear twist as seen in $\mathrm{H} \alpha$. Measurements of the transverse magnetic fields made at Crimea show that the magnetic field is generally twisted down in the photosphere as well.

Bumba: There is evidence about the spiral structures around the sunspots in the chromosphere as has been pointed out by Dr Stenflo. But you may observe such structures around sunspots very rarely in the photosphere. We have succeeded in obtaining a series of good photographs of three regular sunspots 
demonstrating the development of such spiral organization of penumbral fibrils. Studying these series of photographs we come to the conclusion that the observed spiral structures are possibly not connected with the kinematics of the discrete sunspots. On the other hand Knoška's investigation of sunspot rotation shows clearly that many sunspots rotate around their vertical axis, although the greater part of them rotate in the same direction in both solar hemispheres.

Stix: There are indirect indications of polar field reversals prior to the one of $1957 / 58$, e.g. poleward migration of filament zones, or numbers of polar formulae. But even if we forget these, there was still another observed polar field reversal occurring in 1971/72, and I wonder whether you or maybe Dr Howard could comment on the reality of this last reversal.

Howard: There is no doubt that the polar field of the Sun reversed around the time of the last solar maximum - in 1969 and 1972. My paper in Solar Physics gives more details of this.

Giovanelli: But your own results (Solar Phys. 25, 5 (1972); 38, 59, 283 (1974)) show that the polar fields vary irregularly, with little correspondence between north and south. Under these circumstances it is rather difficult to speak confidently about reversals of polar fields being associated with solar maxima; to me, it seems so far that they have been quite irregular. Observations will be needed over a much longer period to establish the pattern.

Vandakurov: I want to note that the problem of turbulent diffusion in the presence of a magnetic field may also be considered from the point of view of stability theory. The estimate turbulent transport characteristics, we use the hypothesis of Kadomtsev and Pogutse which was cited in my paper, published recently in Solar Physics. This seems to favour usually quoted values of the turbulent diffusion. It should be emphasized however that this approach is valid only if the magnetic configuration is stationary. But as far as the convection zone with large convective motion is concerned, the fulfilment of the stationary condition is rather a difficult problem.

Chvojková: Wouldn't it be possible to join these two theories? Dr Stix's report, for example, has finished with the evolution of a poloidal field, while Babcock had begun by a poloidal field which became amplified by winding around the Sun due to differential rotation. Babcock's mechanism could, perhaps, additionally amplify the field given by Stix. It seems that in most phenomena the joint model would be very similar to Piddington's. 